Chapman University

Chapman University Digital Commons

Physical Therapy Faculty Articles and Research

Physical Therapy

2011

\title{
An Approach for Identifying Gait Events Using Wavelet Denoising Technique and Single Wireless IMU
}

Rahul Soangra

ChapmanUniversity, soangra@chapman.edu

Thurmon Lockhart

Virginia Tech

Nathalie Van de Berge

Gent University

Follow this and additional works at: https://digitalcommons.chapman.edu/pt_articles

Part of the Movement and Mind-Body Therapies Commons, Musculoskeletal System Commons, Orthotics and Prosthetics Commons, and the Physical Therapy Commons

\section{Recommended Citation}

Soangra R, Lockhart TE, Van de Berge N. An approach for identifying gait events using wavelet denoising technique and single wireless IMU. Proc Hum Factors Ergon Soc Annu Meet. 2011;55:1990-1994. doi: 10.1177/1071181311551415

This Conference Proceeding is brought to you for free and open access by the Physical Therapy at Chapman University Digital Commons. It has been accepted for inclusion in Physical Therapy Faculty Articles and Research by an authorized administrator of Chapman University Digital Commons. For more information, please contact laughtin@chapman.edu. 


\section{An Approach for Identifying Gait Events Using Wavelet Denoising Technique and Single Wireless IMU}

\section{Comments}

This article was originally published in Proceedings of the Human Factors and Ergonomics Society Annual Meeting, volume 55, in 2011. DOI:10.1177/1071181311551415

\section{Copyright}

Not subject to U.S. copyright restrictions. 


\title{
An approach for identifying gait events using wavelet denoising technique and single wireless IMU
}

\author{
Rahul Soangra ${ }^{2}$, Thurmon E. Lockhart ${ }^{1-3}$, Nathalie Van de Berge ${ }^{3}$ \\ 1. Industrial and Systems Engineering, Virginia Tech ${ }^{1}$, 2. School of Biomedical Engineering \& \\ Sciences, Virginia Tech Wake Forest University 3. Industrial Management Engineering, Gent \\ University
}

A new approach is proposed to identify gait events in non-laboratory environments with a single inertial measurement unit (IMU) embedded inside shoe. The aim of our work is to develop a useful clinical tool for monitoring individuals walking disability and detect specific pathological gait patterns. Temporal parameters of gait are determined by classification of accelerations and angular velocities. Wavelets denoising of IMU signals allows for an important amount of information that is exploited in different manners for event identification. It was found that wavelet denoising enhanced specific turning points which could effectively identify gait events. The method is verified by comparing the results of video-based motion capture system and force plates as conventional standards. This portable gait-monitoring system allows for versatile application beyond gait laboratory.

\section{Introduction:}

In the past year 2009, about 2.2 million nonfatal fall injuries were reported among older adults and were treated in emergency departments and more than 581,000 of these patients were hospitalized(CDC, 2010). Also, in the year 2007, over 18,000 older adults died from fall injuries (CDC, 2010). A recent survey by Liberty mutual research institute for safety ranks falls as the second leading cause of disabling injury and number of falls remain at same level since last decade and cost about $\$ 8.37$ billion each year. Falls due to locomotive impairment cause a serious hazards to elderly, which has a lately become a public research interest (Maki, Holliday, \& Topper, 1991; Ohtaki, Sagawa, \& Inooka, 2001). Normal and pathological gait analysis in laboratory surroundings are performed using expensive instruments such as optokinetic measurement systems for measuring lower extremity movements and force plates for measuring ground reaction forces. However, these measures are costly and time consuming, rely on patient recall and may not accurately reflect functional ability in the patient's home environment and thus have limited validity. In order to prevent falls, there is a serious need to identify an individual's personal risk of falls in the same environment and situations in which they actually live. In the past decade, several researchers have used accelerometers and gyroscopes to detect gait events and spatiotemporal characteristics (Aminian,
Najafi, Bula, Leyvraz, \& Robert, 2002; Auvinet et al., 2002; Pappas, Popovic, Keller, Dietz, \& Morari, 2001; Sabatini, Martelloni, Scapellato, \& Cavallo, 2005; Tong \& Granat, 1999). An accelerometry based analysis has proved to investigate individual characteristics of locomotion in daily living environments (Aminian et al., 1999; Aminian, Robert, Jequier, \& Schutz, 1995). In the present study, we have tried to achieve this goal by using a wireless IMU embedded inside the shoe sole. The nature of sensor inside shoe enables measurements in an natural setting during normal walking in daily life. Information obtained represents subjects capability as well as what a subject actually does while walking.

The morphology of IMU signal has been used for recognizing kinematic events (Aminian, et al., 1999; Lau \& Tong, 2008; Pappas, et al., 2001). This identification step gives a full picture and detailed information about the temporal gait parameters like heel off (HO), heel strike (HS) and toe off (TO). In order to support the clinical decision making, IMU signal must be clearly represented and filtered, to remove out all noises and artifacts from the signal. IMU signals are non-stationary and need denoising, and an efficient technique for non-stationary signal processing is wavelet transform. The wavelet transform can be used as a decomposition of a signal in the time-frequency scale plane. Until now all previous work in the field of gait characteristics and its analysis using accelerometers and 
gyroscopes have involved digital filters (FIR or IIR) and adaptive filter methods to remove noise. However, digital filters and adaptive filter methods can be applied to signals whose statistical characteristics are stationary. Another major interest of this work is to shed some light to the IMU signal noise reduction using wavelet transform.

Wavelets give a superior performance in IMU signal denoising due to properties such as sparsity and multiresolution structure. Discrete wavelet Transform (DWT) for a particular choice of mother wavelet yields an efficient representation of a particular IMU signal class. Signals in the class are well-described using a few large transform coefficients. Also the unstructured noise which is present in accelerometer and gyroscope signals fails to be efficiently represented by discrete wavelet transform (DWT). Due to the orthogonality of the DWT, such noise sequences makes on average, equal contributions to all transform coefficients. Any given noise sequence is expected to yield many small-valued transform coefficients. These small valued details are left out and only amplitude is considered for signal reconstruction. This idea is used in our work as a means of denoising signals from IMU.

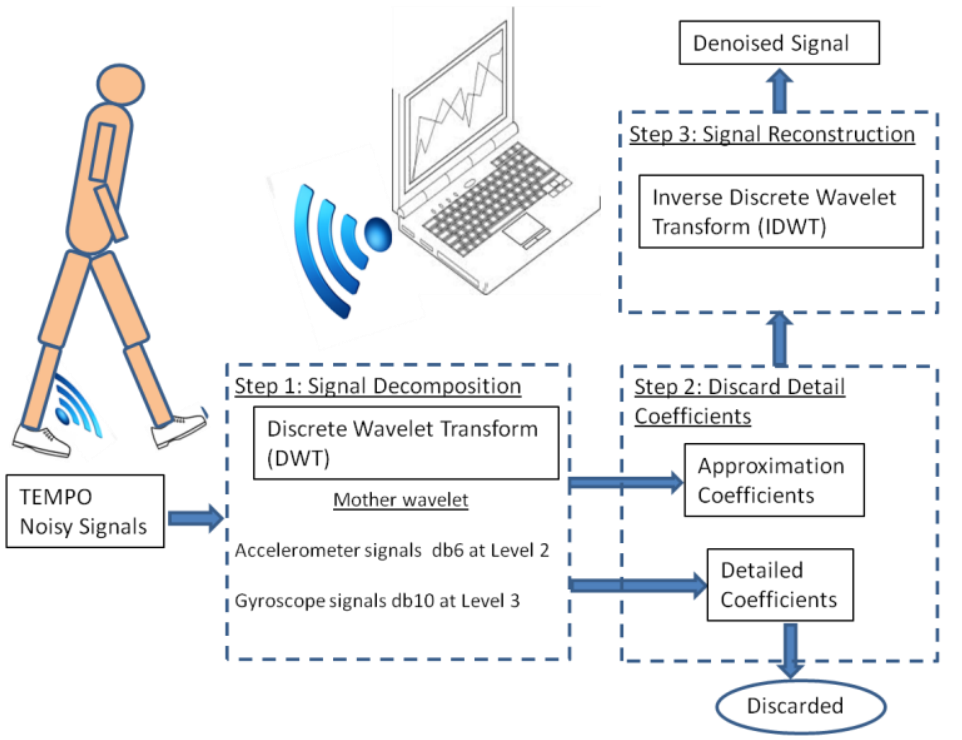

Figure 1: Collection of TEMPO signals and proposed denoising algorithm.

Materials and Methods : Right shoe sole was cut out and alginate mold was poured in to the sole to make a tight fix for the sensor in two pair of shoes of different sizes (Figure 2). IMU used is TEMPO 3.1 which is manufactured in collaborative research with inertia team in UVA (Barth, Hanson, Powell, \& Lach, 2009). It consist of MMA7261QT tri-axial accelerometers and IDG-300 ( $\mathrm{x}$ and y plane gyroscope) and ADXRS300 as z-plane uniaxial gyroscope. The data acquisition was carried using a bluetooth adapter and computer through a custom built LabView VI (Barth, et al., 2009).

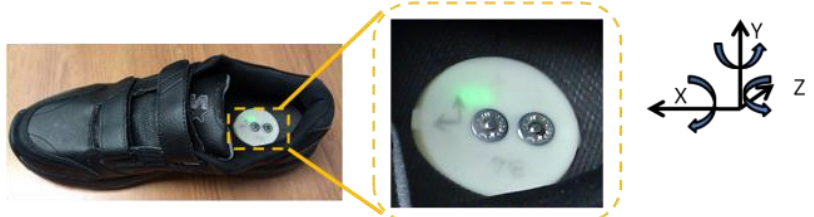

Figure 2: TEMPO IMU sensor embedded inside shoe sole and alignment of its axis's.

Signal Processing: The wavelet transform is used as a signal decomposition and inverse discrete wavelet transform as signal reconstruction technique in the time-frequency scale plane. In wavelet transform, the original signal is transformed using a selected mother wavelet. Using pilot hit and trial testing of various mother wavelets we figured out that daubechies family wavelet ( $\mathrm{db} 10)$ were beneficial in highlighting gait events. In discrete case like ours the wavelet transform is modified to filter bank tree structure using the decomposition / reconstruction at different decomposition levels for accelerometer and gyroscope signals. Basically, decomposition of the signal into the basis of wavelet functions implies the computation of the inner products between the signal and the prototype functions, leading to a set of coefficients called wavelet coefficients. The signal can consequently be reconstructed as a linear combination of the prototype functions weighted by the wavelet coefficients. But here in our denoising protocol we have set all detail coefficients to zero and carried on reconstruction of the signal (Figure 1). Thus, only the approximation coefficients are used in an inverse wavelet transformation to reconstruct the data-set. The mother wavelet $\psi(\mathrm{t})$ is defined as

$$
\psi(t)=\sqrt{2} \sum_{k=-\infty}^{\infty} g(k) \phi(2 t-k)
$$

Where the wavelet coefficients

$$
\begin{gathered}
g(k)=(-1)^{k} h(1-k) \quad \text { and the scaling } \\
\varphi(t)=\sqrt{2} \sum_{k=-\infty}^{\infty} h(k) \varphi(2 t-k)
\end{gathered}
$$

function $\varphi$ 
Where $h(k)$ are the scaling coefficients. The wavelet transform represents the decomposition of a function into a family of wavelet functions.

Subjects : A total of ten subjects participated in our complete study. Experiment part I consisted of development and verification of five participant gait event detection algorithm with motion analysis system in Locomotion Research Laboratory, situated at Virginia Tech. Five subjects (two females and three males, $26 \pm 3$ years) volunteered for this study. The experimental procedures were explained to all participants and informed consent was obtained individually before the experiment. Only healthy college students volunteered for this study, although there was no exclusion criteria but our study was limited to participants with two shoe sizes. Two TEMPO (IMU) nodes were used in the experiment. The TEMPO node inside the shoe was started and each subject was asked to don the shoes with a TEMPO node inside the right shoe sole. Another TEMPO sensor was mounted on the right wrist of the subjects. Five infra-red markers were placed on right toe, right heel, right lateral malleolus and right lateral epicondyle and right ulnar stylus bony landmarks. Two force platforms (BERTEC \#K80102, Type 45550-08, Bertec Corporation, $\mathrm{OH} 43212$, USA) were embedded in the middle of the walkway. A six-camera ProReflex system (Qualysis AB, Gothenburg, Sweden) and the force plates were used to obtain kinematics and kinetics of the lower extremity during gait on the walking platform. Participants were asked to tap quickly near the right wrist and start walking on a $(15 \mathrm{~m} X 1.5 \mathrm{~m})$ walkway. This was conducted in order to synchronize the TEMPO signals with the Ulnar stylus infra red marker which were temporally synchronized with Force plates by a custom built LabView VI.
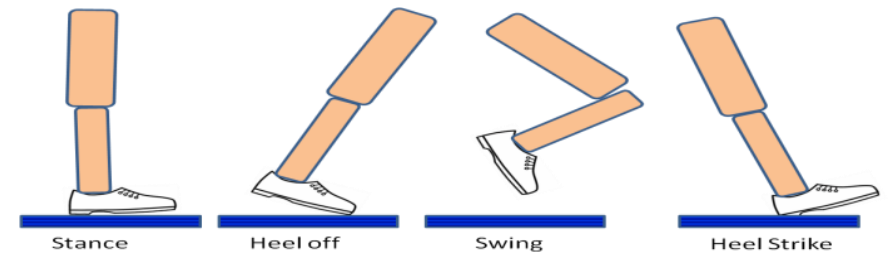

Figure 3: Four phases of gait: stance, heel off, swing, and heel strike.

The sampling frequency for marker data and force plates and TEMPO was set to $120 \mathrm{~Hz}$. Zero phase digital filtering was performed on marker position data and force platform data using low pass butterworth filter. The cut-off frequency chosen was $6 \mathrm{~Hz}$. In experiment part II we recruited five more participants (four males and one female, 19.6 \pm 0.5 years). The algorithm developed was further tested on these five participants and gait parameters were determined. All the kinematics and kinetic data were collected using the camera and force plate settings described above to calculate various temporal gait parameters.

Denoising method: When we decompose a data set using wavelets, we use filters that act as averaging filters and others that produce details which are some of the resulting wavelet coefficients corresponding to details in the data set. If the details are small, they might be omitted without substantially affecting the main features of the data set. In our proposed algorithm for denoising we have ignored the idea of thresholding, and set all detail coefficients to zero. Thus only the approximation coefficients are used in an inverse wavelet transformation to reconstruct the data set. Simple three steps of denoising algorithm that used the wavelet transform are Step 1. Calculate the wavelet transform of the noisy signal; Step 2. Discard the noisy wavelet detail coefficients; Step 3. Compute the inverse transform using the approximation coefficients (Figure 1). Accelerometer signals were decomposed by mother wavelet db6 (Daubechies family(Daubechies, 1988)) at level 2 and gyroscope signals were decomposed using db10 at level 3. A matlab routine was created using mallat's algorithm of discrete wavelet transform(Mallat, 1989).

Gait Event Identification: Gait cycle is divided into four gait phases: stance, heel off, swing and heel-strike (Pappas, et al., 2001) and along with these phases toe off event was detected (Figure 3). The transitions between these phases was identified by denoising IMU signals with various wavelets and signal decomposition to different levels after off-line processing and testing of numerous experimental data (Figure 4). Heel strike events were identified using denoised acc- $\mathrm{x}$ signals from the TEMPO (Figure 5) when acc- $x$ is greater than $1 \mathrm{~g}$ and the slope of the signal was greater than 0.45 units, the point was identified as heel strike. These criteria for identification of heel strike were found 
reasonable since there is sudden decline in foot acceleration in sensitive $\mathrm{X}$ direction of IMU (axis's shown in figure 2) during heel strike and slope of 0.45 is a measure of minimum rate at which the acceleration in this direction drops.

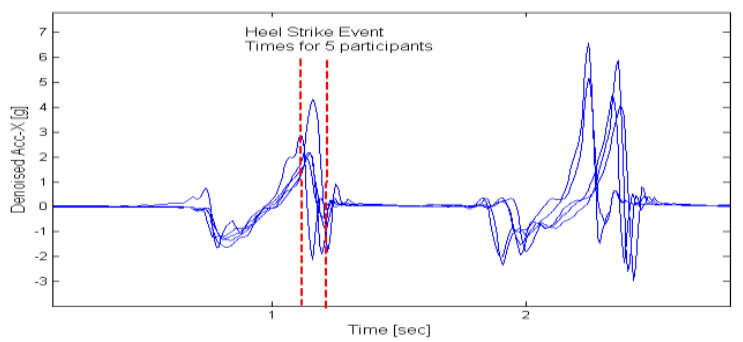

Figure 4: Display of denoised Acc-X signals during walking from five participants with their trials superimposed. These trials have been aligned to uniform initiation time marker (see the text for details). Time scale is identical for each trace.

There is always some component of gravity influencing the accelerometer sensitive axis's and thus magnitude greater than $1 \mathrm{~g}$ seemed reasonable. Stance phase: Stance phase is the interval from heel strike to heel off. Heel off phase: The start of heel off phase is the instant after heel strike when the inclination of the heel with respect to ground exceeds a given threshold angle of $2^{\circ}$ degrees obtained by integration of denoised gyro-y signals from the segmented data set between two consecutive heel strikes with integration start point as when gyro-y signal is one standard deviation below its mean of the data segment (Figure 7). Previous study by Pappas and his group used a similar criteria of threshold angle of $3^{\circ}$ but their placement of gyroscope was at heel and outside shoe (Pappas, et al., 2001). The heel off phase continues till the toe off event. Swing phase: Swing phase starts after the toe off event occurs. Toe off event is the peak angular velocity (gyro-Y) between the two consecutive heel strikes after the heel off event.

Results and Discussion: In proper interpretation of gait pathology, there is a requirement for quantification and comparison with established normal limits. Temporal parameters of gait such as stance time and swing time as percentages of gait cycle enable data comparison, since individuals walk at varying speeds. The presented methodology is successful in denoising IMU signals to an extent which play an important role in event identification.

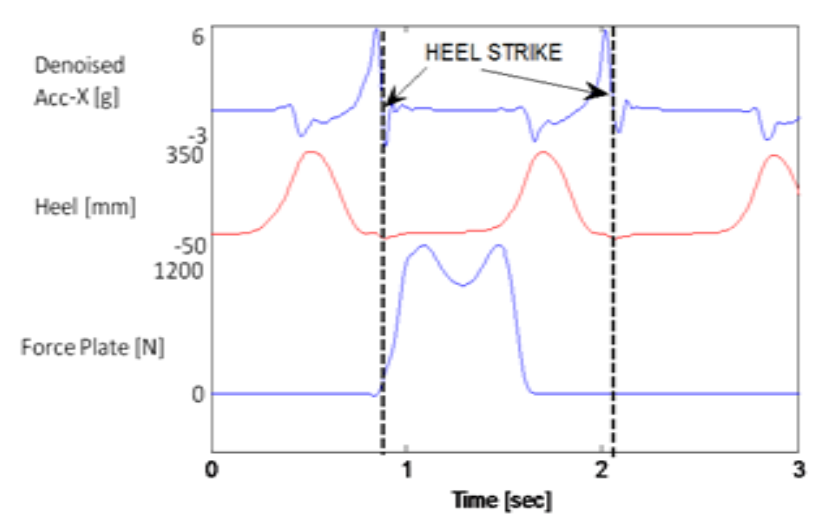

Figure 5: Heel strike event identification using denoised Acc-X and verification using heel marker and forceplate.

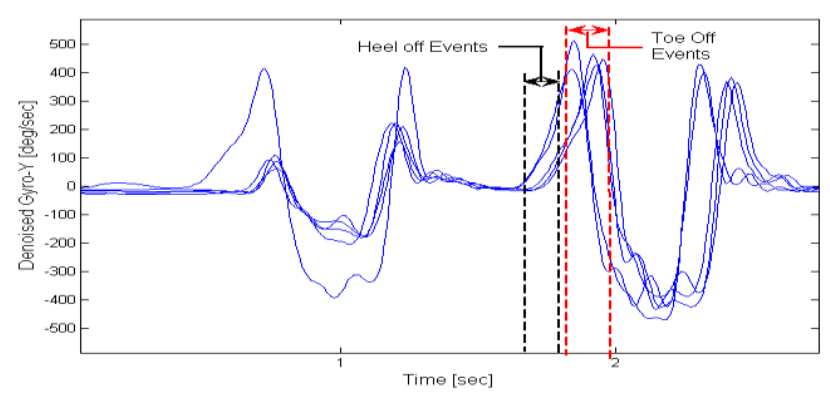

Figure 6: Display of denoised Gyro-Y signals during walking from five participants with their trials superimposed, with trials aligned.

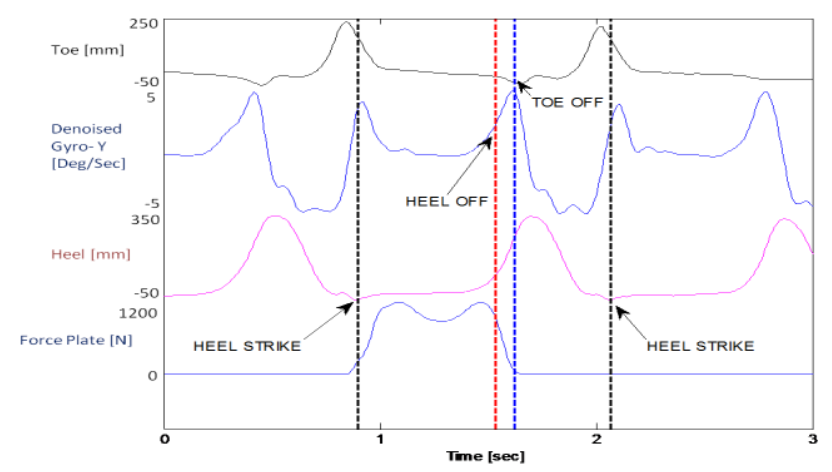

Figure 7: Toe marker, heel marker and forceplate data provide a means of comparison of detected heel off and toe off events.

The results of our experiment II showed that our algorithm is not only reliable but are also robust during walking (Table 1). An advantage of using IMU is that the data can be collected continuously and the subject is not required to stay in $3 \mathrm{D}$ space as such to be detected by camera. 


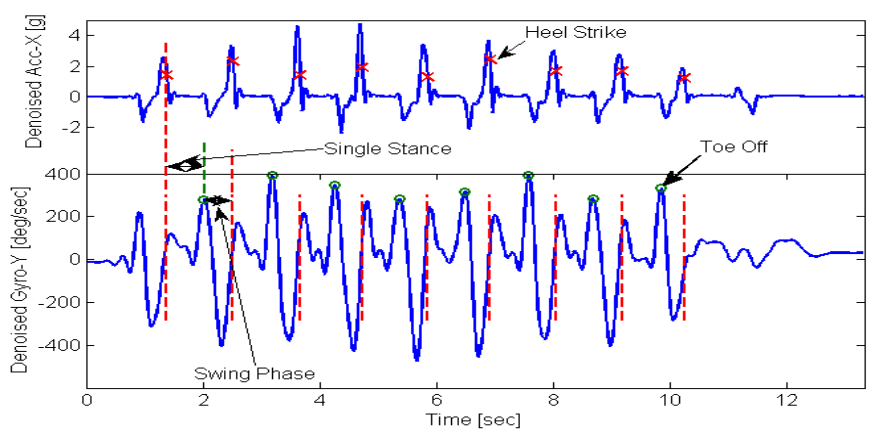

Figure 8: Heel strike and Toe off event detection of a participant walking on $15 \mathrm{~m}$ track and further computation of right leg swing and stance time.

Thus, our study establishes particular approach to fit IMU signals that has non stationary clinical gait information. Also, experimental results show that our noise reduction algorithm exhibits much better performance in gait event identification which is comparable to that in laboratory environment (Table 1).Table 1: Comparsion of temporal parameters of gait.

\begin{tabular}{|c|c|c|c|c|c|c|}
\hline \multirow[b]{2}{*}{$\begin{array}{l}\text { Partic } \\
\text { ipant }\end{array}$} & \multicolumn{3}{|c|}{ Camera System } & \multicolumn{3}{|c|}{ Shoe Embedded TEMPO } \\
\hline & $\begin{array}{l}\text { Right } \\
\text { Leg } \\
\text { Stance } \\
\text { Time } \\
\text { (sec) }\end{array}$ & $\begin{array}{l}\text { Right } \\
\text { Leg } \\
\text { Swing } \\
\text { Time } \\
\text { (sec) }\end{array}$ & $\begin{array}{l}\text { Gait } \\
\text { Cycle } \\
(\mathrm{sec})\end{array}$ & $\begin{array}{l}\text { Right } \\
\text { Leg } \\
\text { Stance } \\
\text { Time } \\
\text { (sec) }\end{array}$ & $\begin{array}{l}\text { Right } \\
\text { Leg } \\
\text { Swing } \\
\text { Time } \\
\text { (sec) }\end{array}$ & $\begin{array}{l}\text { Gait } \\
\text { Cycle } \\
(\mathrm{sec})\end{array}$ \\
\hline 1 & $\begin{array}{l}0.5687 \\
\pm 0.081\end{array}$ & $\begin{array}{l}0.4292 \\
\pm 0.017\end{array}$ & $\begin{array}{l}0.9458 \\
\pm 0.076\end{array}$ & $\begin{array}{l}0.6333 \\
\pm 0.027\end{array}$ & $\begin{array}{l}0.4778 \\
\pm 0.009\end{array}$ & $\begin{array}{l}1.1111 \\
\pm 0.041\end{array}$ \\
\hline 2 & $\begin{array}{l}0.5625 \\
\pm 0.024\end{array}$ & $\begin{array}{l}0.3917 \\
\pm 0.023\end{array}$ & $\begin{array}{l}0.9667 \\
\pm 0.047\end{array}$ & $\begin{array}{l}0.5917 \\
\pm 0.021\end{array}$ & $\begin{array}{l}0.4056 \\
\pm 0.019\end{array}$ & $\begin{array}{l}1 \\
\pm 0.016\end{array}$ \\
\hline 3 & $\begin{array}{l}0.7146 \\
\pm 0.024\end{array}$ & $\begin{array}{l}0.4042 \\
\pm 0.017\end{array}$ & $\begin{array}{l}1.1292 \\
\pm 0.005\end{array}$ & $\begin{array}{l}0.6917 \\
\pm 0.031\end{array}$ & $\begin{array}{l}0.5278 \\
\pm 0.009\end{array}$ & $\begin{array}{l}1.2278 \\
\pm 0.034\end{array}$ \\
\hline 4 & $\begin{array}{l}0.7125 \\
\pm 0.049\end{array}$ & $\begin{array}{l}0.4458 \\
\pm 0.029\end{array}$ & $\begin{array}{l}1.1750 \\
\pm 0.047\end{array}$ & $\begin{array}{r}0.6500 \\
\pm 0.013\end{array}$ & $\begin{array}{l}0.5333 \\
\pm 0.016\end{array}$ & $\begin{array}{l}1.1833 \\
\pm 0.028\end{array}$ \\
\hline 5 & $\begin{array}{l}0.7104 \\
\pm 0.008\end{array}$ & $\begin{array}{l}0.4375 \\
\pm 0.041\end{array}$ & $\begin{array}{l}1.1417 \\
\pm 0.047\end{array}$ & $\begin{array}{l}0.6083 \\
\pm 0.034\end{array}$ & $\begin{array}{l}0.4889 \\
\pm 0.048\end{array}$ & $\begin{array}{l}1.0833 \\
\pm 0.033\end{array}$ \\
\hline
\end{tabular}

This work would lay foundation in clinical assessment of normal and pathological gait in nonlaboratory environments.

Acknowledgements The research is supported by National Science Foundation NSF-CBET-0756058 (VT)/0756645 (UVA) - Collaborative Research. Virginia Tech IRB was approved for this study.

\section{References}

Aminian, K., Najafi, B., Bula, C., Leyvraz, P. F., \& Robert, P. (2002). Spatio-temporal parameters of gait measured by an ambulatory system using miniature gyroscopes. Journal of Biomechanics, 35(5), 689-699. doi: Pii S0021-9290(02)00008-8
Aminian, K., Rezakhanlou, K., De Andres, E., Fritsch, C., Leyvraz, P. F., \& Robert, P. (1999). Temporal feature estimation during walking using miniature accelerometers: an analysis of gait improvement after hip arthroplasty. Medical \& Biological Engineering \& Computing, 37(6), 686-691.

Aminian, K., Robert, P., Jequier, E., \& Schutz, Y. (1995). Incline, Speed, and Distance Assessment during Unconstrained Walking. Medicine and Science in Sports and Exercise, 27(2), 226-234.

Auvinet, B., Berrut, G., Touzard, C., Moutel, L., Collet, N., Chaleil, D., \& Barrey, E. (2002). Reference data for normal subjects obtained with an accelerometric device. Gait \& Posture, 16(2), 124-134. doi: Pii S0966-6362(01)00203-X

Barth, A. T., Hanson, M. A., Powell, H. C., \& Lach, J. (2009). TEMPO 3.1: A Body Area Sensor Network Platform for Continuous Movement Assessment. Paper presented at the Sixth International Workshop on Wearable and Implantable Body Sensor Networks Berkeley, CA

CDC. (2010). Centers for Disease Control and Prevention. Center for Injury Prevention and Control Retrieved from http://www.cdc.gov/injury/wisqars/.

Daubechies, I. (1988). Orthonormal Bases of Compactly Supported Wavelets. Communications on Pure and Applied Mathematics, 41(7), 909-996.

Lau, H. Y., \& Tong, K. Y. (2008). The reliability of using accelerometer and gyroscope for gait event identification on persons with dropped foot. Gait \& Posture, 27(2), 248-257. doi: DOI 10.1016/j.gaitpost.2007.03.018

Maki, B. E., Holliday, P. J., \& Topper, A. K. (1991). Fear of Falling and Postural Performance in the Elderly. Journals of Gerontology, 46(4), M123-M131.

Mallat, S. G. (1989). A Theory for Multiresolution Signal Decomposition - the Wavelet Representation. Ieee Transactions on Pattern Analysis and Machine Intelligence, 11(7), 674-693.

Ohtaki, Y., Sagawa, K., \& Inooka, H. (2001). A method for gait analysis in a daily living environment by bodymounted instruments. Jsme International Journal Series C-Mechanical Systems Machine Elements and Manufacturing, 44(4), 1125-1132.

Pappas, I. P. I., Popovic, M. R., Keller, T., Dietz, V., \& Morari, M. (2001). A reliable gait phase detection system. Ieee Transactions on Neural Systems and Rehabilitation Engineering, 9(2), 113-125.

Sabatini, A. M., Martelloni, C., Scapellato, S., \& Cavallo, F. (2005). Assessment of walking features from foot inertial sensing. Ieee Transactions on Biomedical Engineering, 52(3), 486-494. doi: Doi 10.1109/Tbme.2004.840727

Tong, K. Y., \& Granat, M. H. (1999). A practical gait analysis system using gyroscopes. Medical Engineering \& Physics, 21(2), 87-94. 